\title{
Regulation of Juvenile Hormone on Summer Diapause of Geleruca daurica and Its Pathway Analysis
}

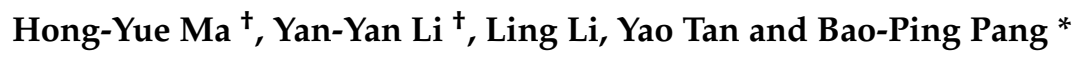 \\ Research Center for Grassland Entomology, Inner Mongolia Agricultural University, Hohhot 010020, China; \\ plmmhy1111@163.com (H.-Y.M.); lyy4455@163.com (Y.-Y.L.); 1ling@imau.edu.cn (L.L.); \\ 850310.tanhuaf4@163.com (Y.T.) \\ * Correspondence: pangbp@imau.edu.cn; Tel.: +86-181-4710-8339 \\ + The same contribution.
}

check for

updates

Citation: Ma, H.-Y.; Li, Y.-Y.; Li, L.; Tan, Y.; Pang, B.-P. Regulation of Juvenile Hormone on Summer Diapause of Geleruca daurica and Its Pathway Analysis. Insects 2021, 12, 237. https://doi.org/10.3390/ insects12030237

Received: 29 January 2021

Accepted: 8 March 2021

Published: 11 March 2021

Publisher's Note: MDPI stays neutral with regard to jurisdictional claims in published maps and institutional affiliations.

Copyright: (c) 2021 by the authors. Licensee MDPI, Basel, Switzerland. This article is an open access article distributed under the terms and conditions of the Creative Commons Attribution (CC BY) license (https:/ / creativecommons.org/licenses/by/ $4.0 /)$.
Simple Summary: Diapause is an arrestment state in development, and plays an important role in life history in insects. It has been thought that a lack in juvenile hormone $(\mathrm{JH})$ results in reproductive diapause occurring at the adult stage. However, we do not fully know about the underlying molecular mechanism. In this study, we proved that the topical application of a JH analog methoprene caused the changes at the transcriptional levels of a great number of genes, inhibited lipid accumulation, and finally delayed the adults entering diapause. Therefore, JH signaling plays an important role in regulating reproductive diapause of G. daurica, a new pest with great outbreaks in Inner Mongolia.

Abstract: Juvenile hormone $(\mathrm{JH})$ signaling plays an important role in regulation of reproductive diapause in insects. However, we have little understanding of the effect of JH on gene expression at the transcriptome level in diapause. Galeruca daurica is a new pest in the Inner Mongolia grasslands with obligatory summer diapause in the adult stage. Topical application of a JH analog methoprene at the pre-diapause stage delayed the adults entering diapause and inhibited lipid accumulation whereas it did not during diapause. Using Illumina sequencing technology and bioinformatics tools, 54 and 138 differentially expressed genes (DEGs) were detected at 1 and $2 \mathrm{~d}$ after treatment, respectively. The KEGG analysis showed that the DEGs were mainly enriched in the metabolism pathways. qRT-PCR analysis indicated that methoprene promoted the expression of genes encoding vitellogenin, fork head transcription factor and Krüppel homolog 1, whereas suppressed the expression of genes encoding juvenile hormone-binding protein, juvenile hormone esterase, juvenile hormone acid methyltransferase, juvenile hormone epoxide hydrolase and fatty acid synthase 2 . These results indicate that $\mathrm{JH}$ signaling plays an important role in regulating reproductive diapause of G. daurica.

Keywords: Galeruca daurica; juvenile hormone; methoprene; diapause; RNA-Seq

\section{Introduction}

Diapause is an adaptive strategy of insects to overcome adverse environmental seasons and ensure population continuity [1]. Diapause can occur at any developmental stage, such as embryonic, larval, pupal and adult stages, however, for any given species, diapause usually occurs at a single stage [2]. Adult diapause is also known as reproductive diapause, and its central feature is the suppression of reproductive functions such as maturing of ovaries and male accessory glands, and mating activity [3]. Adult diapause can be usually attributed to the absence of juvenile hormone (JH) [4]. $\mathrm{JH}$ is a family of sesquiterpenoids, and is known to play a critical role in regulating insect metamorphosis, reproduction, and diapause [5,6]. The molecular basis of the JH signaling pathway has been recently unveiled [7-11]. Many studies have suggested that exogenous $\mathrm{JH}$ analogue (JHA) would mimic the action of JH and likely act directly on the $\mathrm{JH}$ receptors as a substitute for $\mathrm{JH}$ [12]. JHAs have been effectively used to understand the mechanism of $\mathrm{JH}$ action. Topical applications of $\mathrm{JH}$ and several analogues 
one day after the initiation of diapause could break diapause 8-10 days later, whereas $\mathrm{JH}$ application during pre-diapause only delayed diapause in Leptinotarsa decemlineata [13]. Since then, many studies have reported that the application of exogenous JH or JHA can terminate adult diapause and induce ovarian development or oviposition in a number of beetle species [14]. The exposure to JHA methoprene promoted the development of ovaries and terminated reproductive diapause in Melinda pusilla [15] and Polygonia c-aureum [16]. Lipids provide an important energy reserve for diapausing insects, and understanding the molecular mechanisms involved in lipid accumulation during diapause preparation is indispensable to understand the metabolic processes regulating diapause in insects [17]. FASs are highly conserved and crucial genes in the fatty acid biosynthetic pathway [18]. JHA treatment repressed the expression of FAS2 in C. bowringi, and knockdown of FAS2 by RNAi suppressed lipid accumulation [19]. However, up to now, the above studies have focused on facultative diapause, and the molecular basis of this hormonal regulation on reproductive diapause has not been fully understood [20]. To the best of our knowledge, it has been not reported how $\mathrm{JH}$ or JHA influences the gene expression in reproductive diapause at a transcriptome level.

Galeruca daurica (Joannis) (Coleoptera-Chrysomelidae) has become one of the most destructive pests on the grasslands of Inner Mongolia since its sudden outbreak in 2009, and has caused severe economic losses and grassland degradation [21]. This pest is an univoltine species with obligatory summer diapause in the adult stage. Overwintering eggs start to hatch in mid-April. From late May to early June, after newly-emerged adults feed for about one week, the beetles begin to aggregate under grass, cow dung and stone, and enter diapause. About three months later, adults terminate diapause and reactivate, and lay eggs in autumn [22]. Our previous studies suggested that the JH signaling pathway might play a crucial role in regulating diapause in G. daurica $[23,24]$. In this study, in order to clarify the effect of $\mathrm{JH}$ on obligatory reproductive diapause and its global gene regulatory cascades, we used the next-generation high-throughput sequencing technology (RNA-Seq) to analyze the transcriptional changes in response to topical JHA treatment in the G. daurica adults, measured the effects of JHA on the total lipid content and diapause rate of G. daurica, and finally compared the expression levels of the eight JH-related genes after JHA application by qRT-PCR. The results revealed a great number of JH target genes and metabolic pathways. Therefore, our study provides a solid foundation for further elucidation of the regulatory cascades in JH-controlled reproductive diapause.

\section{Materials and Methods}

\subsection{Insect Rearing}

Eggs were originally collected from the Xilingguole grasslands of Inner Mongolia, China, on 15 April 2019. The insects were reared in the Research Center for Grassland Entomology, Inner Mongolia Agricultural University. Eggs were incubated in an incubator (temperature: $25 \pm 1{ }^{\circ} \mathrm{C}$; $\mathrm{RH}: 80 \pm 5 \%$; photoperiod: $14 \mathrm{~h}$ light: $10 \mathrm{~h}$ dark). Insects were reared individually in Petri dishes $(9.0 \mathrm{~cm}$ in diameter and $1.5 \mathrm{~cm}$ in height) lined with moist filter papers under natural conditions after larval emergence. Allium mongolium was provided as food once a day.

\subsection{JHA Treatment}

JHA methoprene (CAS-number: 40596-69-8; purity: 98.0\%; MedChemExpress, China) was diluted to $25 \mathrm{mg} / \mathrm{mL}$ concentrations using dimethyl sulfoxide (DMSO) and stored at $-80^{\circ} \mathrm{C}$ as a stock solution, and then diluted to 1.0 and $2.5 \mu \mathrm{g} / \mu \mathrm{L}$ working concentrations with normal saline. A DMSO solution diluted with normal saline was used as a negative control, and untreated adults as blank controls. Two microlitres of control solution or treatment liquor was injected into the adult abdomens using a microsyringe.

\subsection{RNA Extraction and cDNA Synthesis}

Total RNA was extracted using RNAiso plus reagent (TaKaRa. Dalian, China) according to the manufacturer's instructions. RNA concentration and purity were determined 
using a NanoPhotometer ${ }^{\circledR}$ P330 microspectrophotometer (Implen, Munich, Germany). The integrity of RNA was detected using electrophoresis in 1\% agarose gel. The RNA was treated with DNAase to eliminate residual genomic DNA and then complementary DNA (cDNA) was synthesized for qPCR using the PrimeScript ${ }^{\circledR}$ RT Reagent Kit with gDNA Eraser (TaKaRa, Dalian, China) and stored at $-20^{\circ} \mathrm{C}$ until use.

\subsection{Transcriptome Sequencing, Assembly and Bioinformatics Analysis}

For transcriptome analysis, two microlitres of methoprene $(2.5 \mu \mathrm{g} / \mu \mathrm{L})$ was topically applied to the adults at 3 days after eclosion. After JHA treatment, individuals were sampled at $1(\mathrm{Ta})$ and 2 days $(\mathrm{Tb})$ post-injection, respectively, and individuals treated with carrier solution served as control groups ( $\mathrm{CKa}, 1$ day post-injection; and $\mathrm{CKb}, 2$ days post-injection). Three biological replicates of each treatment with at least 10 individuals ( 5 females and 5 males) per replication were performed. Samples were sent to Biomarker Technologies Co, LTD (Beijing, China) for transcriptome sequencing. The sequencing libraries were generated using NEBNext ${ }^{\circledR}$ Ultra $^{\mathrm{TM}}$ RNA Library Prep Kit for Illumina ${ }^{\circledR}$ (NEB, Ipswich, MA, USA) following manufacturer's recommendations. To select cDNA fragments of preferentially $240 \mathrm{bp}$ in length, the library fragments were purified with the AMPure XP system (Beckman Coulter, Beverly, MA, USA). Library quality was assessed on the Agilent Bioanalyzer 2100 system. The library preparations were sequenced on the Illumina Hiseq 6000 platform and paired-end reads were generated. Clean data were obtained by removing reads containing adapter, ploy-N and low-quality read from raw data. At the same time, Q20, Q30, GC-content, and sequence duplication levels of the clean data were calculated. De novo assembly of RNA-Seq was performed based on the left.fq and right.fq using Trinity [25] with min_kmer_cov set to 2 by default and all other parameters set default. The annotation of assembled sequences was performed by BLASTn and BLASTx (E-values $\leq 1 \times 10^{-5}$ ) based on the following databases- $\mathrm{Nr}$ (NCBI on-redundant protein sequences), Nt (NCBI non-redundant nucleotide sequences), Pfam (Protein family), KOG/COG (Clusters of Orthologous Groups of proteins), Swiss-Prot (A manually annotated and reviewed protein sequence database), KO (KEGG Ortholog database) and GO (Gene Ontology).

Transcript expression level was estimated in combination with RSEM (RNA-Seq by Expectation-Maximization) [26]. The FPKM (Fragments Per Kilobase of transcript per Million mapped reads) value was used to represent the expression level of corresponding transcripts. Differential expression analysis was performed using the DESeq2 [27]. The Benjamini and Hochberg approaches were used to adjust $p$ values (false discovery rate, FDR). Genes with FDR $<0.05$ and FC (Fold change) $\geq 2$ were assigned as differentially expressed genes (DEGs). Gene Ontology (GO) enrichment analysis of DEGs was performed by GOseq R packages based on Wallenius non-central hyper-geometric distribution [28]. KOBAS software was used to test the statistical enrichment pathways of DEGs in the Kyoto Encyclopedia of Genes and Genomes (KEGG) [29].

\subsection{Quantitative Real-Time PCR}

To analyze the expression of the JH-induced genes, the treated insects were collected at $1,2,4,6,8,10$, and 12 days after JHA application. Quantitative real-time PCR (qRTPCR) was used to determine the expression levels of 8 genes related to the JH pathway after JHA treatment, including JHBP (juvenile hormone binding protein), JHE (juvenile hormone esterase), JHAMT (juvenile hormone acid methyltransferase), JHEH (juvenile hormone epoxide hydrolase), $V g$ (vitellogenin), FOXO (fork head transcription factor), $K r-h 1$ (Krüppel homolog 1), and FAS2 (fatty acid synthase 2). These 8 genes were selected based on our RNA-Seq database assembled in this study and documents [5,19]. The gene-specific primers were designed using an online qRT-PCR primer design tool (http://www.ncbi.nlm.nih.gov/tools/primer-blast/ (accessed on 15 September 2020)) based on our RNA-Seq data of G. daurica (Table S1). The qRT-PCR reaction was conducted using the BRYT Green ${ }^{\circledR}$ dye kit (Promega, Fitchburg, WI, USA) and the FTC-3000P Real- 
Time Quantitative Thermal Cycler systems (Funglyn Biotech, Richmond Hill, ON, Canada). The succinate dehydrogenase complex (SDHA) was used as the reference gene [21]. qRTPCR reactions were performed in $20 \mu \mathrm{L}$ mixture, which consisted of $2 \mu \mathrm{L}$ of cDNA, $0.4 \mu \mathrm{L}$ of forward and reverse primer $(10 \mu \mathrm{mol} / \mathrm{L}), 10 \mu \mathrm{L}$ GoTaq ${ }^{\circledR}$ qPCR Master Mix, and $7.2 \mu \mathrm{L}$ of RNase-free $\mathrm{H}_{2} \mathrm{O}$. All reactions used the following amplification conditions-initial denaturation at $95{ }^{\circ} \mathrm{C}$ for $10 \mathrm{~min}$, followed by 40 cycles at $95{ }^{\circ} \mathrm{C}$ for $15 \mathrm{~s}, 60{ }^{\circ} \mathrm{C}$ for $1 \mathrm{~min}$, and $72{ }^{\circ} \mathrm{C}$ for $15 \mathrm{~s}$. The qRT-PCR data were analyzed by the $2^{-\Delta \Delta \mathrm{Ct}}$ method [30] based on three biological replicates and four technical replicates. Each biological replicate included 3 females and 3 males.

\subsection{Total Lipid Content Measurement}

The total lipid content was determined according to the chloroform-methyl alcohol method [31]. Briefly, the treated insects were placed in a centrifuge tube, and dried in an oven at $60{ }^{\circ} \mathrm{C}$ for 3 days, weighed to measure dry mass (DM), then ground in $1 \mathrm{~mL}$ chloroform-methanol (2:1) solution and then centrifugation occurred. The lean dry mass (LDM) was measured after placed in an oven at $60^{\circ} \mathrm{C}$ and baked to constant weight. The fat content $(\mathrm{FC})$ was calculated by $\mathrm{FC}=(\mathrm{DM}-\mathrm{DM}) / \mathrm{LDM}$. Three independent biological replicates were performed for each treatment, and each replicate included 5 females and 5 males and one individual was tested each time. Therefore, the FC value of each replicate was calculated as a mean of 10 values (individuals), and the FC value of each treatment was calculated as a mean of three replicates (30 individuals).

\subsection{Behavioral and Developmental Observation}

To investigate the effects of JHA (methoprene) on the development of G. daurica adults, the topical applications of methoprene of two concentrations $(2.5$ and $1.0 \mu \mathrm{g} / \mu \mathrm{L})$ were performed on the adults at 3, 5, and 15 days post-eclosion, respectively. Each treatment included three biological replicates and each replicate contained 25 males and 25 females. The beetles were observed twice a day for feeding behavior and we recorded the number of feeding adults every day after treatment. When adults began to stop feeding continuously, diapause entry was determined, because our previous observation indicated that adults started to stop feeding about one week after eclosion and enter diapause with sudden decreasing in respiration intensity [22,23].

\subsection{Statistical Analysis}

Differences in the expression levels of each target gene after JHA treatments were analyzed using one-way analysis of variance (ANOVA) followed by Tukey's Honestly Significant Difference (HSD) tests $(p<0.05)$. All datasets were analyzed using SPSS 20.0 software. All data were presented as the mean \pm SE (standard error).

\section{Results}

\subsection{Effects of JHA on the Development of G. daurica Adults}

The results showed that JHA treatments at 3 and 5 days post-eclosion affected significantly the development of adults but the 15-day treatment did not (Figure 1). When JHA applications were conducted at 3 days post-eclosion, the diapause rates of JHA $(2.5 \mu \mathrm{g} / \mu \mathrm{L})$, JHA $(1.0 \mu \mathrm{g} / \mu \mathrm{L})$, solvent control, and blank control reached $50 \%$ at $12.90,11.10,8.34$, and 7.84 days post-eclosion, respectively. With exposure to 2.5 and $1.0 \mu \mathrm{g} / \mu \mathrm{L}$ JHA, the diapause rates at $50 \%$ were delayed by 4.56 , and 2.76 days, respectively, compared with the solvent controls (Figure 1A). When JHA applications were conducted at 5 days post-eclosion, the diapause rates of JHA $(2.5 \mu \mathrm{g} / \mu \mathrm{L})$, JHA $(1.0 \mu \mathrm{g} / \mu \mathrm{L})$, solvent control, and blank control reached to $50 \%$ at $14.30,12.90,8.88$, and 7.72 days post-eclosion, respectively. With exposure to 2.5 and $1.0 \mu \mathrm{g} / \mu \mathrm{L} \mathrm{JHA}$, the diapause rates at $50 \%$ was delayed by 5.42 and 4.02 days, respectively, compared with the solvent control (Figure 1B). However, when JHA applications were conducted at 15 days post-eclosion, all treated individuals still kept diapause. The results indicated that JHA application significantly postponed diapause 
initiation when the treatment was performed at the pre-diapause stage ( 3 and 5 days after eclosion), whereas it did not affect diapause when the treatment was conducted during diapause (15 days after eclosion) in G. daurica.
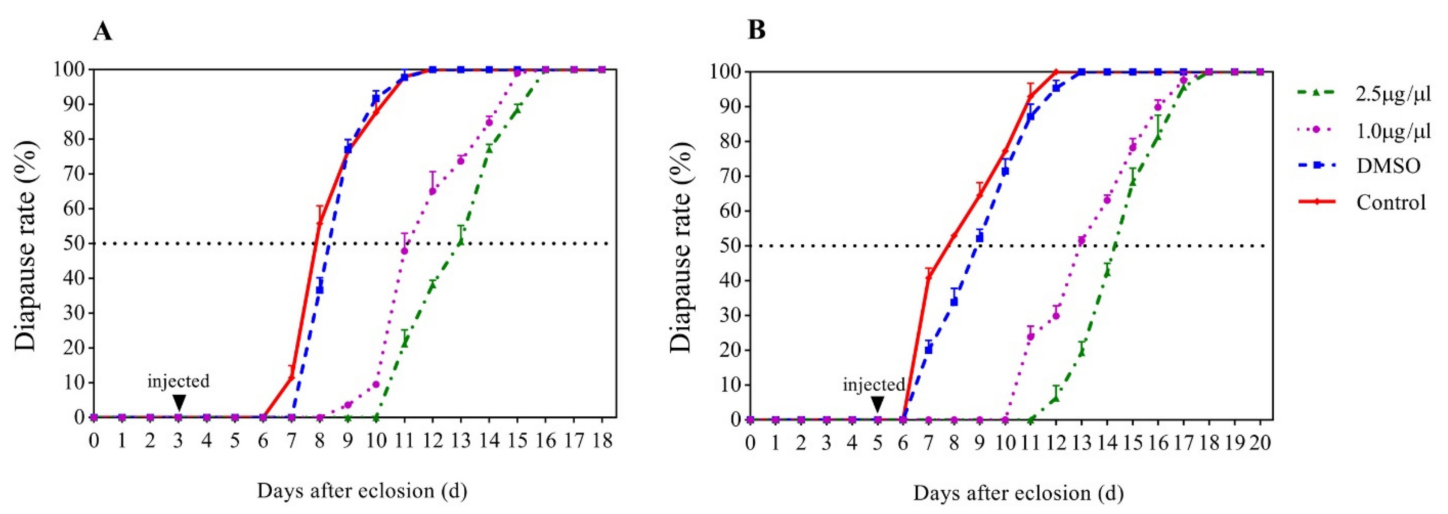

Figure 1. Effects of juvenile hormone analogue (JHA) JHA on diapause rates of G. daurica adults. (A) JHA application at 3 days post-eclosion. (B) JHA application at 5 days post-eclosion.

\subsection{Effects of JHA on the Total Lipid Content in G. daurica}

The effects of JHA treatments at 3 and 5 days post-eclosion on the total lipid content are shown in Figure 2. The result showed that JHA treatments affected significantly the lipid content in the G. daurica adults. When JHA was applied at 3 days post-eclosion, the lipid contents in JHA treatment were significantly less than those in the DMSO controls from 1 to 6 days after treatment (Figure 2A). When JHA was applied at 5 days post-eclosion, the lipid contents in JHA treatment were significantly less than those in the DMSO controls from 1 to 4 days after treatment (Figure 2B). These results showed that JHA application at the pre-diapause stage could inhibit the lipid accumulation in the G. daurica adults, and this inhibitory effect lasted for at least 4 days.
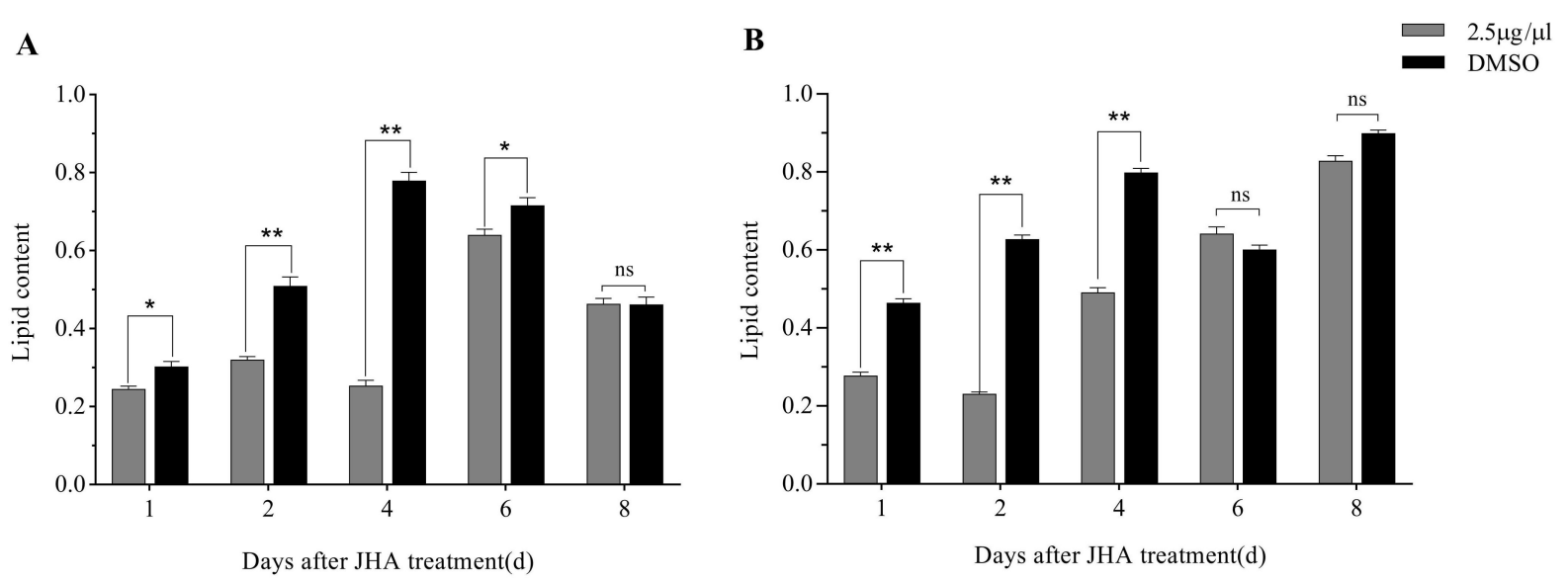

Figure 2. Effects of JHA on the total lipid content of G. daurica adults. (A) JHA application 3 days after adult eclosion. (B) JHA application 5 days after adult eclosion. The data are shown as the mean \pm SE. * and ** indicate significant difference at $p<0.05$ and $p<0.01$, respectively, and 'ns' means no significant difference at $p<0.05$ (Student's $t$-test).

\subsection{Transcriptome Changes in Response to JHA Application}

To reveal the molecular mechanism of JH signaling in diapause regulation at the transcriptional level in G. daurica, RNA-Seq was performed for the adults after JHA treatments. A total of 87,236 unigenes with an average length of $838.12 \mathrm{bp}$ and an N50 of $1677 \mathrm{bp}$ were obtained by Trinity assembly from 12 samples (CKa1, CKa2, CKa3, CKb1, CKb2, CKb3, Ta1, Ta2, Ta3, Tb1, Tb2, Tb3) (Table S2). Compared with the control (CKa), 54 DEGs 
(34 up-regulated and 20 down-regulated) were obtained one day after JHA application, and compared with the control (CKb), 138 DEGs (48 up-regulated and 90 down-regulated) were found at 2 days after JHA application. Among them, 11 common DEGs (four upregulated and seven down-regulated) were identified (Figure 3 and Table 1). Finally, all of the DEGs were characterized by BLAST to investigate the homologous protein-coding genes annotated in the Nr database (Table S3).

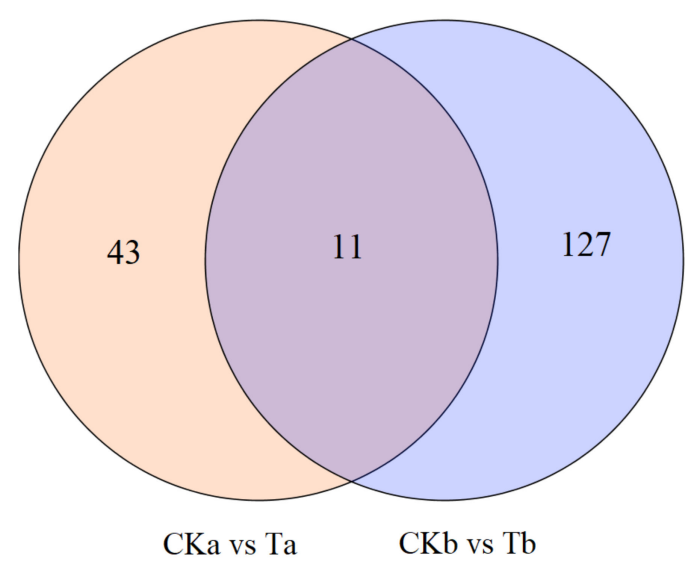

Figure 3. Venn diagram of differentially expressed genes after JHA application. $\mathrm{CKa}$ and $\mathrm{CKb}$ (controls) -1 and 2 days post-injection with carrier solution, respectively; Ta and $\mathrm{Tb}$ (treatments) -1 and 2 days post-injection with methoprene $(2.5 \mu \mathrm{g} / \mu \mathrm{L})$, respectively.

Table 1. Common 11 differentially expressed genes in $\mathrm{CKa}$ vs. Ta and $\mathrm{CKb}$ vs. $\mathrm{Tb}$ comparisons.

\begin{tabular}{|c|c|c|c|c|c|c|c|}
\hline \multirow{2}{*}{ DEG_ID } & \multicolumn{2}{|c|}{ Cka vs. Ta } & \multicolumn{4}{|c|}{ CKb vs. Tb } & \multirow{2}{*}{ Nr_Annotation } \\
\hline & FDR & $\log 2 \mathrm{FC}$ & Regulated & FDR & $\log 2 \mathrm{FC}$ & Regulated & \\
\hline c85279.graph_c0 & $2.37 \times 10^{-13}$ & 2.575 & up & $2.92 \times 10^{-2}$ & 1.303 & up & vitellogenin-like \\
\hline c82613.graph_c0 & $6.25 \times 10^{-6}$ & 1.369 & up & $1.19 \times 10^{-3}$ & 1.380 & up & fatty acid synthase-like \\
\hline c74827.graph_c0 & $3.39 \times 10^{-3}$ & 1.336 & up & $5.83 \times 10^{-3}$ & 1.558 & up & uncharacterized protein LOC111513065 \\
\hline c71191.graph_c0 & $1.61 \times 10^{-5}$ & 1.272 & up & $4.84 \times 10^{-3}$ & 1.228 & up & probable pseudouridine-5\&apos;-phosphatase \\
\hline c69643.graph_c0 & $8.60 \times 10^{-3}$ & -1.007 & down & $7.39 \times 10^{-4}$ & -1.295 & down & retinoid-inducible serine carboxypeptidase-like \\
\hline c37561.graph_c0 & $2.11 \times 10^{-6}$ & -1.034 & down & $1.46 \times 10^{-9}$ & -1.617 & down & PREDICTED: crustapain-like \\
\hline c64926.graph_c0 & $5.25 \times 10^{-3}$ & -1.065 & down & $7.01 \times 10^{-11}$ & -1.777 & down & - \\
\hline c84589.graph_c0 & $9.74 \times 10^{-5}$ & -1.160 & down & $1.64 \times 10^{-5}$ & -1.526 & down & protein msta-like \\
\hline c72112.graph_c0 & $1.80 \times 10^{-5}$ & -1.360 & down & $6.99 \times 10^{-30}$ & -2.327 & down & facilitated trehalose transporter Tret1-like \\
\hline c73343.graph_c0 & $2.44 \times 10^{-3}$ & -1.365 & down & $4.20 \times 10^{-2}$ & -1.330 & down & - \\
\hline c82886.graph_c0 & $3.61 \times 10^{-6}$ & -1.401 & down & $4.31 \times 10^{-2}$ & -1.235 & down & nose resistant to fluoxetine protein 6-like isoform $\mathrm{X} 1$ \\
\hline
\end{tabular}

To analyze the putative molecular mechanisms of JHA effects on reproductive diapause, the annotated DEGs were subjected to the KEGG and GO enrichment analysis. For the CKa vs. Ta comparison, eight DEGs were enriched in the 18 KEGG pathways, among which nine pathways $(50 \%)$ belonged to metabolism pathways, and the main enrichment pathways included apoptosis, steroid biosynthesis, glycosaminoglycan degradation, glycolysis/gluconeogenesis, and the lysosome. (Figure $4 \mathrm{~A}$ and Table $\mathrm{S4}$ ). For the $\mathrm{CKb}$ vs. Tb comparison, $24 \mathrm{DEGs}$ were enriched in the 32 KEGG pathways, among which 13 pathways (40.6\%) belonged to metabolism pathways, and the main enrichment pathways contained herpes simplex infection, ascorbate and aldarate metabolism, fatty acid biosynthesis, drug metabolism — other enzymes, and ABC transporters (Figure 4B and Table S4). These results indicated that the JHA treatment affected mainly the metabolism pathways in the G. daurica adults.

The GO enrichment analysis indicated that for the CKa vs. Ta comparison, 20, nine, and three terms were significantly enriched in the biological process, cellular component, and molecular function, respectively. The top three most significantly terms were the oxidation-reduction process, nucleic acid metabolic process, and developmental process for the biological process; ribonucleoprotein complex, cytoplasm, and cell periphery for the cellular component; and nucleic acid binding, oxidoreductase activity, and catalytic activity for the molecular function. For the $\mathrm{CKb}$ vs. Tb comparison, 32, 12, and six terms 
were significantly enriched in the biological process, cellular component, and molecular function, respectively. The top three most significantly enriched terms were translation, ribosome assembly, and the oxidation-reduction process for the biological process; ribosome, intracellular organelle lumen, and ribosomal subunit for the cellular component; and nucleic acid binding, oxidoreductase activity, and structural constituent of cuticle for the molecular function (Table S5).

A

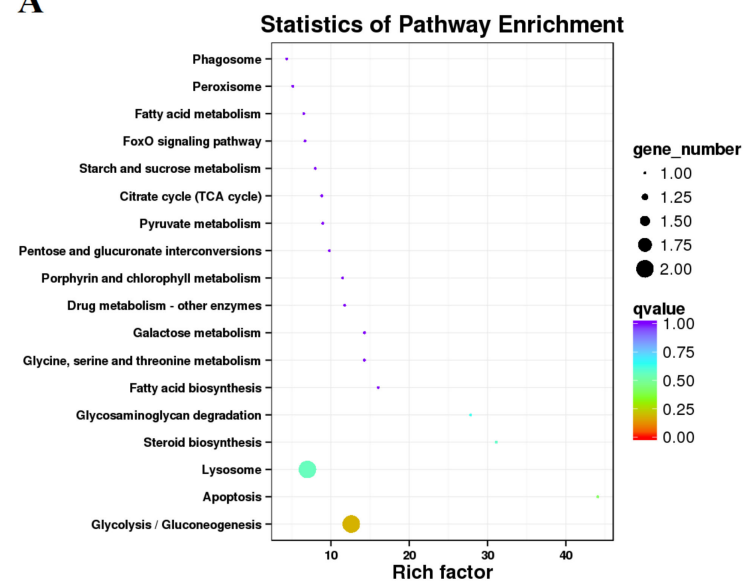

B



Figure 4. Enriched KEGG pathways for differentially expressed genes after JHA application. (A) One day after JHA application. (B) Two days after JHA application.

\subsection{Effects of JHA Application on JH Signaling Pathway-Related Gene Expression}

To reveal the molecular mechanism of JHA inhibiting reproductive diapause, the relative expression profiles of the eight JH signaling-related DEGs, including JHAMT, JHBP, $J H E$, JHEH, $\mathrm{Kr}-h 1, F O X O, V g$, and FAS2, were further analyzed by qRT-PCR at more time points after JHA treatment. We first examined the effects of JHA application at 3 days post adult eclosion. qRT-PCR analysis showed that both two doses $(2.5$ and $1.0 \mu \mathrm{g} / \mu \mathrm{L}$ methoprene) of JHA application influenced significantly the expression of all the other seven genes except for JHAMT ( $p<0.05)$ (Figure 5). Compared with the DMSO-treated or untreated controls, JHBP was down-regulated during the first 2 days after treatment but up-regulated at 4 and 6 days after treatment (Figure 5A). JHA treatments decreased the JHE expression from 1 to 6 days at a low dose $(1.0 \mu \mathrm{g} / \mu \mathrm{L})$ or 8 days at a high dose $(2.5 \mu \mathrm{g} / \mu \mathrm{L})$ after treatment (Figure 5C). The $V g$ expression was up-regulated from 1 to 4 days $(1.0 \mu \mathrm{g} / \mu \mathrm{L})$ or 6 days $(2.5 \mu \mathrm{g} / \mu \mathrm{L})$ after treatment, and the stimulating effect at a dose of $2.5 \mu \mathrm{g} / \mu \mathrm{L}$ was much higher than at a dose of $1.0 \mu \mathrm{g} / \mu \mathrm{L}$ (Figure 5E). JHA application down-regulated significantly the expression of FAS2 only at a high dose of $2.5 \mu \mathrm{g} / \mu \mathrm{L}$. The high-dose treatment induced the expression of FOXO from 1 to 6 days after treatment whereas the low dose did only at the first day (Figure $5 \mathrm{G}$ ). $K r$ - $h 1$ showed a similar response to methoprene treatment to FOXO (Figure $5 \mathrm{H}$ ). However, the $J H E H$ expression was reduced during the first 6 days after treatment with a low dose of $1.0 \mu \mathrm{g} / \mu \mathrm{L}$ whereas this inhibiting effect lasted for 2 days after treatment with a high dose of $2.5 \mu \mathrm{g} / \mu \mathrm{L}$ (Figure 5D).

Figure 6 showed that the effects of JHA application at 5 days post adult eclosion were similar to those at 3 days post adult eclosion except for JHAMT, which was down-regulated during 2 days after treatment at a high dose of $2.5 \mu \mathrm{g} / \mu \mathrm{L}$. In conclusion, JHA methoprene could promote the expression of $V g, F O X O$ and $K r-h 1$ while suppressing the expression of JHBP, JHAMT, JHE, JHEH and FAS2, and the action strength increased with the JHA dose increasing except for JHEH. However, JHA application during diapause (at 15 days post adult eclosion) did not significantly affect the expression of the above eight genes, in keeping with no influence on the diapause rates of the G. daurica adults (Figure S1). 

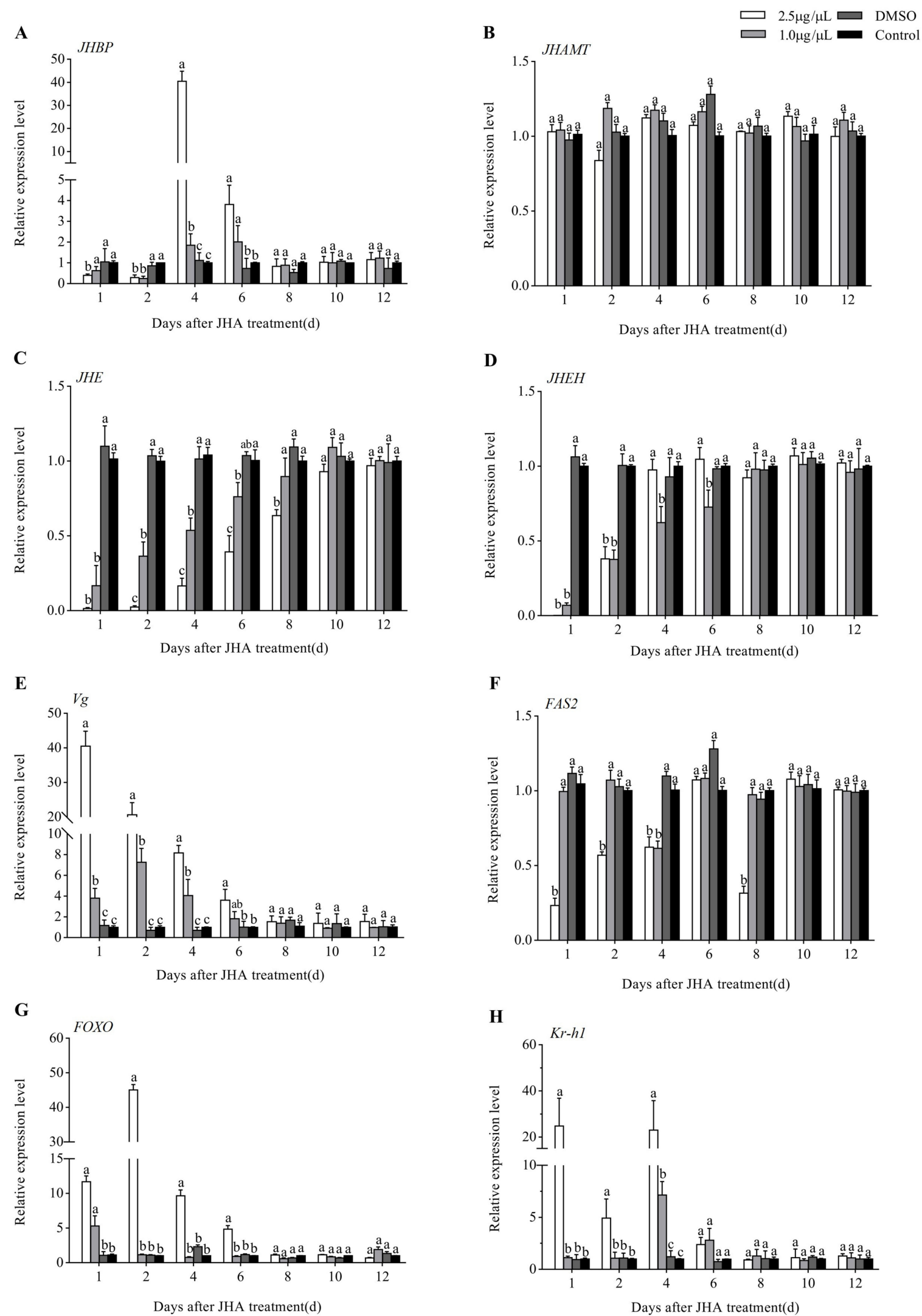

Figure 5. Expression profiles of $8 \mathrm{JH}$ signaling-related genes after JHA application at 3 days post-eclosion. Different letters above the columns indicate significant difference among different treatments at the same time at $p<0.05$ (Tukey's HSD test). (A): JHBP; (B): JHAMP; (C): JHE; (D): JHEH; (E): Vg; (F): FAS2; (G): FOXO; (H): Kr-h1. 

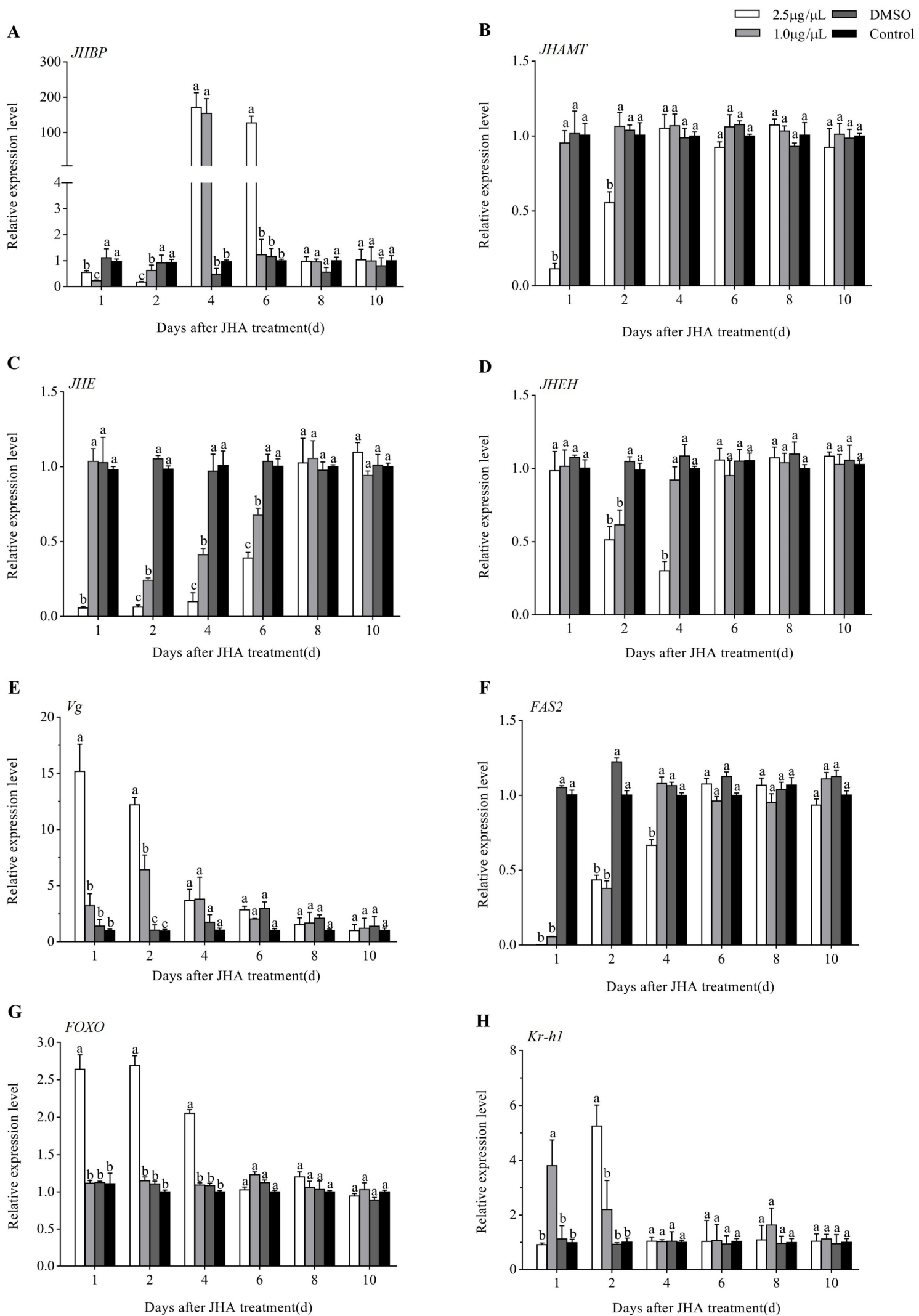

Figure 6. Expression profiles of $8 \mathrm{JH}$ signaling-related genes after JHA application at 5 days post-eclosion. Different letters above the columns indicate significant difference among different treatments at the same time at $p<0.05$ (Tukey's HSD test). (A): JHBP; (B): JHAMP; (C): JHE; (D): JHEH; (E): Vg; (F): FAS2; (G): FOXO; (H): Kr-h1. 


\section{Discussion}

A great deal of literature shows that the lack of $\mathrm{JH}$ can lead to reproductive diapause in insects [4]. Schooneveld et al. [13] first reported that the topical application of JH only postponed diapause during pre-diapause of L. decemlineata, but adults during diapause were activated rather easily. JHA treatment (methoprene) could terminate reproductive diapause of M. pusilla [15]. JH treatment restrained diapause in Culex tritaeniorhynchus whereas it promoted the diapausing females to terminate diapause [32]. Hiroyoshi et al. [16] also found that JHA, methoprene, promoted the development of ovaries and of the male accessory glands and simplex, indicating that methoprene could induce the termination of reproductive diapause. However, in this study, JHA treatment in the pre-diapause stage could delay temporarily the G. daurica adults entering diapause but could not break diapause when JHA was applied during diapause. A possible reason is that the other aforementioned insect species are species with facultative diapause whereas diapause in G. daurica belongs to obligatory diapause, which can be usually terminated only after a longer diapause maintenance period.

In the present study, the regulation network of $\mathrm{JH}$ signaling-associated genes in G. daurica was investigated through the transcriptome sequencing after JHA treatment. The results showed that a great number of genes changed at the transcriptional expression level, and these DEGs were enriched in various metabolism pathways, suggesting that JH might function in the regulation of diapause by regulating metabolic processes in G. daurica [33]. Zhu et al. [34] also found that mRNA levels of 16 and 72 genes were enhanced at 3 and $12 \mathrm{~h}$ after $\mathrm{JH}$ treatment using microarray hybridization meanwhile mRNA levels of 33 and 76 genes were significantly suppressed at 3 and $12 \mathrm{~h}$, respectively, in newly emerged adults of Aedes aegypti, and the observed changes could be also induced by the JHAs, methoprene and pyriproxyfen, but not by farnesol.

In this study, the mRNA levels of several key genes involved in JH biosynthesis and degradation processes were found to change after JHA application, such as JHAMT, JHE, $J H E H$, and JHBP. JH titer in insects is regulated by JH biosynthesis and degradation. It has been reported that JHE and JHEH in hemolymph are involved in the JH degradation [34]. We found that topical treatment with JHA methoprene could suppress the expression of $J H E$ and JHEH in G. daurica. Similarly, the JH mimic pyriproxyfen significantly repressed the JHE transcription during the diapause induction phase of L. decemlineata, confirming the important role of JHE in diapause induction of L. decemlineata [35]. In contrast, exogenous JHA treatment promoted the expression of JHE in A. aegypti [34], Heliothis virescens [36], Plutella xylostella [37,38], and Colaphellus bowringi [39]). In Neocaridina davidi, JHEH was up-regulated after JHA treatment and this result could be caused by the hormonal feedback system [40]. JHAMT and JHBP are the most important regulators in the process of $\mathrm{JH}$ biosynthesis and transportation [41,42]. Our qRT-PCR results showed that JHBP was down-regulated in the first two days after JHA treatment, but up-regulated at 4 and 6 days after JHA treatment in G. daurica. However, JHBP was up-regulated following JHA treatment (methoprene) in other insects, such as Omphisa fuscidentalis [43] and Helicoverpa armigera [44]. Additionally, JHA application inhibited the expression of JHAMT in G. daurica, in agreement with the observation in C. bowringi [45]. The above results show that the genes involved in the regulation of JH exhibit obvious differences between various insect species, which indicates that the mechanism of hormone activity in insects is very complicated and the regulation of $\mathrm{JH}$ biosynthesis and degradation is variable. Our work further verifies that the application of exogenous JHA can directly regulate the expression of the JH-signaling pathway genes, and it has great potential in the control of diapause and reproduction of G. daurica.

$\mathrm{Kr}-\mathrm{h} 1$, a zinc finger transcription factor, plays a key downstream role in the JH pathway as a transductor of JH activity [46]. Kr-h1 has been found to be involved in the regulation of metamorphosis, vitellogenesis and oocyte maturation [47-53]. Stagnation in reproduction is a key feature of adult (reproductive) diapause, which implies an arrest in oocyte development for females [4]. Therefore, Kr-h1 may function in regulating adult 
diapause. In this study, JHA treatment was found to induce the expression of $K r-h 1$ and $V g$. Yue et al. [49] also found that JHA treatment (methoprene) significantly increased the expression of $K r-h 1$ and $V g$, and accelerated ovary development in Bactrocera dorsalis. Topical application of JH III stimulated the expression of $K r$-h1 in Sitodiplosis mosellana [54]. The expression of $V g$ induced through treatment with JH or JHA was also found in other insects, such as Blattella germanica [55], Tribolium castaneum [56], Pyrrhocoris apterus [57], and C. bowringi [45].

The insulin signaling pathway and its downstream target, FOXO, play an important role in regulating diapause in some insects, such as Drosophila melanogaster [58], Culex pipiens [59] and Laodelphax striatellus [60]. In the present study, JHA treatment enhanced the expression of $F O X O$ and $V g$, and postponed the initiation of adult diapause. It was also reported that knockdown of FOXO by RNAi inhibited the $V g$ expression in $A$. aegypti [61], and led to a significantly extended duration of summer diapause pupae in Delia antiqua [62]. Nevertheless, topical application of JH III to diapause-destined females of C. pipiens suppressed the FOXO protein expression [63]. This difference might be due to different diapause forms-obligatory summer diapause in G. daurica whereas facultative winter in C. pipiens. We speculate that two transcription factors, $\mathrm{Kr}-\mathrm{h} 1$ and FOXO, might also play important roles in regulating summer diapause of G. daurica adults.

After JHA treatment, many DEGs were enriched in various metabolism pathways which took up $50 \%$ and $40.6 \%$ of all enriched pathways for the DEGs at 1 and 2 days post-JHA treatment, respectively (Table S4). Trehalose transporters (TRETs) participate in transferring trehalose from the fat body into the hemolymph, and they have been also found to play an important role in insect growth and energy metabolism [64]. Although trehalose has been shown to contribute to diapause and reproduction, whether TRETs play roles in these processes remains unclear [65]. In this study, two TRET1 genes and one TRET1 gene were differentially down-regulated 1 and 2 days, respectively, after JHA application. Li et al. [65] also recently reported that JHA induced TRET1b but repressed TRET1 $a$ at transcriptional levels in C. bowringi. Fatty acid biosynthesis and metabolism were common enriched pathways (Figure 4). JHA treatment also inhibited the FAS2 expression, in keeping with the decreasing of the lipid content in G. daurica. Tan et al., got the same results in C. bowringi [19]. These results show that JH signaling plays a regulatory role in the fatty acid biosynthesis and metabolism. However, a transcript (c82613.graph_c0) coding fatty acid synthase-like was up-regulated after JHA application (Table 1). We speculate that JH may have different effects on different FASs, which needs further investigation.

\section{Conclusions}

JHA application postponed diapause initiation and inhibited the lipid accumulation when the treatment was performed at the pre-diapause stage, whereas it did not affect diapause when the treatment was conducted during diapause in G. daurica. JHA application affected mainly the metabolism pathways. JHA could promote the expression of $V g$, FOXO and $K r-h 1$ while it suppresses the expression of JHBP, JHAMT, JHE, JHEH and FAS2, and the action strength increased with the JHA dose increasing. These results show that JH signaling plays an important role in the regulation of reproductive summer diapause in G. daurica.

Supplementary Materials: The following are available online at https:/ /www.mdpi.com/2075-445 0/12/3/237/s1, Figure S1: Expression profiles of $8 \mathrm{JH}$ signaling-related genes after JHA application at 15 days post eclosion, Table S1: Primers for qPCR used in this study, Table S2: Assembly result statistics table, Table S3: The annotation and $p$-value of DEGs in the transcriptomes, Table S4: Enriched KEGG pathways of DEGs, Table S5: Significantly enriched GO terms for the DEGs.

Author Contributions: Conceptualization, H.-Y.M. and Y.-Y.L.; methodology, H.-Y.M.; software, Y.-Y.L. and L.L.; validation, H.-Y.M., Y.-Y.L. and B.-P.P.; formal analysis, H.-Y.M. and Y.-Y.L.; investigation, H.-Y.M. and Y.-Y.L.; resources, Y.T.; data curation, Y.T.; writing-original draft preparation, H.-Y.M. and Y.-Y.L.; writing-review and editing, B.-P.P.; supervision, B.-P.P.; project administration, 
B.-P.P.; funding acquisition, B.-P.P. All authors have read and agreed to the published version of the manuscript.

Funding: This research was funded by the National Natural Science Foundation of China, grant number 31760517.

Institutional Review Board Statement: Not applicable.

Data Availability Statement: Data is contained in the article and Supplementary Materials.

Acknowledgments: We thank Yan-Min Shan (Inner Mongolia Grassland Station, China) for her help to collect insects.

Conflicts of Interest: The authors declare no conflict of interest. The funders had no role in the design of the study; in the collection, analyses, or interpretation of data; in the writing of the manuscript, or in the decision to publish the results.

\section{References}

1. Kostál, V. Eco-physiological phases of insect diapause. J. Insect Physiol. 2006, 52, 113-127. [CrossRef]

2. Denlinger, D.L. Regulation of diapause. Annu. Rev. Entomol. 2002, 47, 93-122. [CrossRef] [PubMed]

3. Hodek, I. Adult diapause in Coleoptera. Psyche 2012, 1-10. [CrossRef]

4. Denlinger, D.L.; Yocum, G.D.; Rinehart, J.P. Hormonal Control of Diapause. In Insect Endocrinology; Gilbert, L.I., Ed.; Elsevier: London, UK, 2012; pp. 430-463.

5. Jindra, M.; Palli, S.R.; Riddiford, L.M. The juvenile hormone signaling pathway in insect development. Annu. Rev. Entomol. 2013, 58, 181-204. [CrossRef]

6. Gujar, H.; Palli, S.R. Juvenile hormone regulation of metamorphosis in the common bed bug, Cimex lectularius. Sci. Rep. 2016, 6, 26092. [CrossRef] [PubMed]

7. Ashok, M.; Turner, C.; Wilson, T.G. Insect juvenile hormone resistance gene homology with the bHLH-PAS family of transcriptional regulators. Proc. Natl. Acad. Sci. USA 1998, 95, 2761-2766. [CrossRef] [PubMed]

8. Minakuchi, C.; Namiki, T.; Shinoda, T. Kruppel homolog 1 an early juvenile hormone-response gene downstream of Methoprenetolerant, mediates its anti-metamorphic action in the red flour beetle Tribolium castaneum. Dev. Biol. 2009, 325, 341-350. [CrossRef] [PubMed]

9. Charles, J.P.; Iwema, T.; Epa, V.C.; Takaki, K.; Rynes, J.; Jindra, M. Ligand-binding properties of a juvenile hormone receptor, Methoprene-tolerant. Proc. Natl. Acad. Sci. USA 2011, 108, 21128-21133. [CrossRef] [PubMed]

10. Jindra, M.; Uhlirova, M.; Charles, J.P.; Smykal, V.; Hill, R.J. Genetic evidence for function of the bHLH-PAS protein Gce/Met as a juvenile hormone receptor. PLoS Genet. 2015, 11, e1005394. [CrossRef]

11. Li, M.; Liu, P.; Wiley, J.D.; Ojani, R.; Bevan, D.R.; Li, J.; Zhu, J. A steroid receptor coactivator acts as the DNA-binding partner of the Methoprene-tolerant protein in regulating juvenile hormone response genes. Mol. Cell. Endocrinol. 2014, $394,47-58$. [CrossRef]

12. Huang, Z.Y.; Lin, S.; Ahn, K. Methoprene does not affect juvenile hormone titers in honey bee (Apis mellifera) workers. Insect Sci. 2018, 25, 235-240. [CrossRef]

13. Schooneveld, H.; Sanchez, A.O.; Wilde, J.D. Juvenile hormone-Induced break and termination of diapause in the Colorado potato beetle. J. Insect Physiol. 1977, 23, 689-696. [CrossRef]

14. Ojima, N.; Ishiguro, S.; An, Y.; Kadosawa, T.; Suzuki, K. Male reproductive maturity and diapause termination in the leaf beetle Gastrophysa atrocyanea. Physiol. Entomol. 2015, 40, 277-283. [CrossRef]

15. Agui, N.; Mihara, M.; Kurahashi, H. Effect of juvenile hormone analogue on ovarian development of the reproductive-diapausing parasitic fly, Melinda pusilla. Jpn. J. Sanit. Zool. 1991, 42, 311-317. [CrossRef]

16. Hiroyoshi, S.; Reddy, G.V.P.; Mitsuhashi, J. Effects of juvenile hormone analogue (methoprene) and 20-hydroxyecdysone on reproduction in Polygonia c-aureum (Lepidoptera: Nymphalidae) in relation to adult diapause. J. Comp. Physiol. A 2017, 203, 635-647. [CrossRef] [PubMed]

17. Hahn, D.A.; Denlinger, D.L. Energetics of insect diapause. Annu. Rev. Entomol. 2011, 56, 103-121. [CrossRef]

18. Visser, B.; Ellers, J. Lack of lipogenesis in parasitoids: A review of physiological mechanisms and evolutionary implications. J. Insect Physiol. 2008, 54, 1315-1322. [CrossRef] [PubMed]

19. Tan, Q.Q.; Liu, W.; Zhu, F.; Lei, C.L.; Wang, X.P. Fatty acid synthase 2 contributes to diapause preparation in a beetle by regulating lipid accumulation and stress tolerance genes expression. Sci. Rep. 2017, 7, 40509. [CrossRef]

20. Gao, Q.; Feng, S.; Liu, W.; Wang, X.P.; Wang, J.L. Research progress in juvenile hormone regulation mechanism in insect reproductive diapause. J. Environ. Entomol. 2018, 40, 988-994.

21. Tan, Y.; Zhou, X.R.; Pang, B.P. Reference gene selection and evaluation for expression analysis using qRT-PCR in Galeruca daurica (Joannis). Bull. Entomol. Res. 2017, 107, 359-368. [CrossRef]

22. Chen, L.; Zhou, X.R.; Gao, L.J.; Tan, Y.; Pang, B.P. Change of carbohydrate, protein and lipid contents in Galeruca daurica (Coleoptera: Chrysomelidae) adults during over summering. Acta Entomol. Sin. 2018, 61, 808-814. 
23. Ma, H.Y.; Zhou, X.R.; Tan, Y.; Pang, B.P. Proteomic analysis of adult Galeruca daurica (Coleoptera: Chrysomelidae) at different stages during summer diapause. Comp. Biochem. Physiol. 2019, 29, 351-357. [CrossRef] [PubMed]

24. Chen, L.; Zhou, X.R.; Tan, Y.; Pang, B.P.; Xinbayin. Cloning and expression profiling of the Galeruca daurica juvenile hormone binding protein gene, GdJHBP. Chin. J. Appl. Entomol. 2020, 57, 623-631.

25. Grabherr, M.G.; Haas, B.J.; Yassour, M.; Levin, J.Z.; Thompson, D.A.; Amit, I.; Xian, A.; Fan, L.; Raychowdhury, R.; Zeng, Q. Full-length transcriptome assembly from RNA-Seq data without a reference genome. Nat. Biotechnol. 2011, 29, 644-652. [CrossRef]

26. Love, M.I.; Huber, W.; Anders, S. Moderated estimation of fold change and dispersion for RNA-seq data with DESeq2. Genome Biol. 2014, 15, 550. [CrossRef] [PubMed]

27. Young, M.D.; Wakefield, M.J.; Smyth, G.K.; Oshlack, A. Gene ontology analysis for RNA-seq: Accounting for selection bias. Genome Biol. 2010, 11, R14. [CrossRef]

28. Ashburner, M.; Ball, C.A.; Blake, J.A.; Botstein, D.; Butler, H.; Cherry, J.M.; Davis, A.P.; Dolinski, K.; Dwight, S.S.; Epig, J.T.; et al. Gene ontology: Tool for the unification of biology. The Gene Ontology Consortium. Nat. Genet. 2000, 25, 25-29. [CrossRef]

29. Mao, X.; Cai, T.; Olyarchuk, J.G.; Wei, L. Automated genome annotation and pathway identification using the KEGG Orthology (KO) as a controlled vocabulary. Bioinformatics 2005, 21, 3787-3793. [CrossRef]

30. Livak, K.J.; Schmittgen, T.D. Analysis of relative gene expression data using real-time quantitative PCR and the $2^{(-\Delta \Delta C t)}$ method. Methods 2001, 25, 402-408. [CrossRef] [PubMed]

31. Colinet, H.; Vernon, P.; Hance, T. Does thermal-related plasticity in size and fat reserves influence supercooling abilities and cold-tolerance in Aphidius colemani (Hymenoptera: Aphidiinae) mummies? J. Therm. Biol. 2007, 32, 374-382. [CrossRef]

32. Wang, Y.; Xue, R.D. Effect of juvenile hormone and light in the initiation and termination of diapause in Culex tritaeniorhynchus. Chin. J. Vector Biol. Control 1994, 5, 410-412.

33. Liu, W.; Tan, Q.Q.; Zhu, L.; Li, Y.; Zhu, F.; Lei, C.L.; Wang, X.P. Absence of juvenile hormone signalling regulates the dynamic expression profiles of nutritional metabolism genes during diapause preparation in the cabbage beetle Colaphellus bowringi. Insect Mol. Biol. 2017, 26, 530-542. [CrossRef] [PubMed]

34. Zhu, J.; Busche, J.M.; Zhang, X. Identification of juvenile hormone target genes in the adult female mosquitoes. Insect Biochem. Mol. Biol. 2010, 40, 23-29. [CrossRef] [PubMed]

35. Vermunt, A.W.M.; Koopmanschap, A.B.; Vlak, J.M.; de Kort, C.A. Expression of the juvenile hormone esterase gene in the Colorado potato beetle, Leptinotarsa decemlineata: Photoperiodic and juvenile hormone analog response. J. Insect Physiol. 1999, 45, 135-142. [CrossRef]

36. Wroblewski, V.J.; Harshman, L.G.; Hanzlik, T.N.; Hammock, B.D. Regulation of juvenile hormone esterase gene expression in the tobacco budworm (Heliothis virescens). Arch. Biochem. Biophys. 1990, 278, 461-466. [CrossRef]

37. Gu, X.; Kumar, S.; Kim, E.; Kim, Y. A whole genome screening and RNA interference identify a juvenile hormone esterase-like gene of the diamondback moth, Plutella xylostella. J. Insect Physiol. 2015, 80, 81-87. [CrossRef]

38. Duan, D.; Zheng, R.; Lin, S.; Chen, Y.; Tian, H.; Zhao, J.; Tian, S.; Wei, H.; Gu, X. Modulation of juvenile hormone esterase gene expression against development of Plutella xylostella (Lepidoptera: Plutellidae). J. Econ. Entomol. 2016, 109, 865-872. [CrossRef]

39. Zhu, L.; Yin, T.Y.; Sun, D.; Liu, W.; Zhu, F.; Lei, C.L.; Wang, X.P. Juvenile hormone regulates the differential expression of putative juvenile hormone esterases via methoprene-tolerant in non-diapause-destined and diapause-destined adult female beetle. Gene 2017, 627, 373-378. [CrossRef]

40. Hu, X.L.; Niu, J.J.; Meng, Q.; Chai, Y.H.; Chan, K.M. Effects of two juvenile hormone analogue insecticides, fenoxycarb and methoprene, on Neocaridina davidi. Environ. Pollut. 2019, 253, 89-99. [CrossRef]

41. Kim, I.H.; Pham, V.; Jablonka, W.; Goodman, W.G.; Ribeiro, J.M.C.; Andersen, J.F. A mosquito hemolymph odorant-binding protein family member specifically binds juvenile hormone. J. Biol. Chem. 2017, 292, 15329-15339. [CrossRef] [PubMed]

42. Nouzova, M.; Michalkova, V.; Ramirez, C.E.; Fernandez-Lima, F.; Noriega, F.G. Inhibition of juvenile hormone synthesis in mosquitoes by the methylation inhibitor 3-deazaneplanocin A (DZNep). Insect Biochem. Mol. Biol. 2019, 113, 103183. [CrossRef] [PubMed]

43. Ritdachyeng, E.; Manaboon, M.; Tobe, S.S.; Singtripop, T. Molecular characterization and gene expression of juvenile hormone binding protein in the bamboo borer, Omphisa fuscidentalis. J. Insect Physiol. 2012, 58, 1493-1501. [CrossRef] [PubMed]

44. Zhang, W.N.; Liang, G.M.; Ma, L.; Jiang, T.; Xiao, H.J. Dissecting the role of juvenile hormone binding protein in response to hormone and starvation in the cotton bollworm, Helicoverpa armigera (Hübner) (Lepidoptera: Noctuidae). J. Econ. Entomol. 2019, 112, 1411-1417. [CrossRef]

45. Liu, W.; Li, Y.; Zhu, L.; Zhu, F.; Lei, C.L.; Wang, X.P. Juvenile hormone facilitates the antagonism between adult reproduction and diapause through the methoprene-tolerant gene in the female Colaphellus bowringi. Insect Biochem. Mol. Biol. 2016, 74, 50-60. [CrossRef]

46. Belles, X.; Santos, C.G. The MEKRE93 (Methoprene tolerant-Krüppel homolog 1-E93) pathway in the regulation of insect metamorphosis, and the homology of the pupal stage. Insect Biochem. Mol. Biol. 2014, 52, 60-68. [CrossRef]

47. Song, J.; Wu, Z.; Wang, Z.; Deng, Z.; Zhou, S. Krüppel-homolog 1 mediates juvenile hormone action to promote vitellogenesis and oocyte maturation in the migratory locust. Insect Biochem. Mol. Biol. 2014, 52, 94-101. [CrossRef] [PubMed]

48. Jiang, J.; Xu, Y.; Lin, X. Role of Broad-Complex (Br) and Krüppel homolog 1 (Kr-h1) in the ovary development of Nilaparvata lugens. Front. Physiol. 2017, 8, 1013. [CrossRef] [PubMed] 
49. Li, K.L.; Yuan, S.Y.; Nanda, S.; Wang, W.X.; Lai, F.X.; Fu, Q.; Wan, P.J. The roles of E93 and Kr-h1 in metamorphosis of Nilaparvata lugens. Front. Physiol. 2018, 9, 1677. [CrossRef] [PubMed]

50. Yue, Y.; Yang, R.L.; Wang, W.P.; Zhou, Q.H.; Chen, E.H.; Yuan, G.R.; Wang, J.J.; Dou, W. Involvement of Met and Kr-h1 in JH-mediated reproduction of female Bactrocera dorsalis (Hendel). Front. Physiol. 2018, 9, 482. [CrossRef]

51. Mao, Y.; Li, Y.; Gao, H.; Lin, X. The direct interaction between E93 and Kr-h1 mediated their antagonistic effect on ovary development of the brown planthopper. Int. J. Mol. Sci. 2019, 20, 2431. [CrossRef]

52. Saha, T.T.; Roy, S.; Pei, G.; Dou, W.; Zou, Z.; Raikhel, A.S. Synergistic action of the transcription factors Krüppel homolog 1 and Hairy in juvenile hormone/Methoprene-tolerant-mediated gene-repression in the mosquito Aedes aegypti. PLoS Genet. 2019, 15, e1008443. [CrossRef]

53. Hu, K.; Tian, P.; Yang, L.; Tang, Y.; Qiu, L.; He, H.; Ding, W.; Li, Y. Molecular characterization of the Krüppel-homolog 1 and its role in ovarian development in Sogatella furcifera (Hemiptera: Delphacidae). Mol. Biol. Rep. 2020, 47, 1099-1106. [CrossRef] [PubMed]

54. Cheng, W.N.; Li, X.J.; Zhao, J.J.; Zhu-Salzman, K. Cloning and characterization of Methoprene-tolerant (Met) and Krüppel homolog 1 (Kr-h1) genes in the wheat blossom midge, Sitodiplosis mosellana. Insect Sci. 2020, 27, 292-303. [CrossRef] [PubMed]

55. Comas, D.; Piulachs, M.D.; Bellés, X. Induction of vitellogenin gene transcription in vitro by juvenile hormone in Blattella germanica. Mol. Cell. Endocrinol. 2001, 183, 93-100. [CrossRef]

56. Parthasarathy, R.; Sun, Z.; Bai, H.; Palli, S.R. Juvenile hormone regulation of vitellogenin synthesis in the red flour beetle, Tribolium castaneum. Insect Biochem. Mol. Biol. 2010, 40, 405-414. [CrossRef]

57. Smykal, V.; Bajgar, A.; Provaznik, J.; Fexova, S.; Buricova, M.; Takaki, K.; Hodkova, M.; Jindra, M.; Dolezel, D. Juvenile hormone signaling during reproduction and development of the linden bug, Pyrrhocoris apterus. Insect Biochem. Mol. Biol. 2014, 45, 69-76. [CrossRef] [PubMed]

58. Junger, M.A.; Rintelen, F.; Stocker, H.; Wasserman, J.D.; Vegh, M.; Radimerski, T.; Greenbery, M.E.; Hafen, E. The Drosophila forkhead transcription factor FOXO mediates the reduction in cell number associated with reduced insulin signaling. J. Biol. 2003, 2, 20. [CrossRef] [PubMed]

59. Sim, C.; Denlinger, D.L. Insulin signaling and FOXO regulate the overwintering diapause of the mosquito Culex pipiens. Proc. Natl. Acad. Sci. USA 2008, 105, 6777-6781. [CrossRef]

60. Yin, Z.J.; Dong, X.L.; Kang, K.; Chen, H.; Dai, X.Y.; Wu, G.A.; Zheng, L.; Zhai, Y.F. FoxO transcription factor regulate hormone mediated signaling on nymphal diapause. Front. Physiol. 2018, 9, 1654. [CrossRef]

61. Hansen, I.A.; Sieglaff, D.H.; Shiao, S.H.; Munro, J.B.; Knox, J.; Raikhel, A.S. The forkhead box gene family of transcription factors of the yellow fever mosquito Aedes aegypti and its role in mosquito reproduction. Am. J. Trop. Med. Hyg. 2006, 75, 262.

62. Peng, Z.Q.; Hao, Y.J. Role of DaFOXO1 in the regulation of superoxide dismutase gene expression and developmental duration summer diapause pupae of Delia antiqua (Diptera: Anthomyiidae). Acta Entomol. Sin. 2019, 62, 1359-1368.

63. Sim, C.; Denlinger, D.L. Juvenile hormone III suppresses forkhead of transcription factor in the fat body and reduces fat accumulation in the diapausing mosquito, Culex pipiens. Insect Mol. Biol. 2013, 22, 1-11. [CrossRef] [PubMed]

64. Kikuta, S.; Hagiwara-Komoda, Y.; Noda, H.; Kikawada, T. A novel member of the trehalose transporter family functions as an $\mathrm{H}^{(+)}$-dependent trehalose transporter in the reabsorption of trehalose in malpighian tubules. Front. Physiol. 2012, 3, 290. [CrossRef] [PubMed]

65. Li, J.X.; Cao, Z.; Guo, S.; Tian, Z.; Liu, W.; Zhu, F.; Wang, X.P. Molecular characterization and functional analysis of two trehalose transporter genes in the cabbage beetle, Colaphellus bowringi. J. Asia Pac. Entomol. 2020, 23, 627-633. [CrossRef] 